\title{
Hybrid Structures of ITO-Nanowire-Embedded ITO Film for the Enhanced Si Photodetectors
}

\author{
Hyunki Kim, ${ }^{1,2}$ Gyeong-Nam Lee, ${ }^{1,2}$ and Joondong Kim $\mathbb{D I D}^{1,2}$ \\ ${ }^{1}$ Department of Electrical Engineering, Incheon National University, 119 Academy Rd., Yeonsu, Incheon 22012, Republic of Korea \\ ${ }^{2}$ Photoelectric and Energy Device Application Lab (PEDAL), Multidisciplinary Core Institute for Future Energies (MCIFE), Incheon \\ National University, 119 Academy Rd., Yeonsu, Incheon 22012, Republic of Korea
}

Correspondence should be addressed to Joondong Kim; joonkim@incheon.ac.kr

Received 31 January 2018; Accepted 14 June 2018; Published 2 July 2018

Academic Editor: Yong Ding

Copyright @ 2018 Hyunki Kim et al. This is an open access article distributed under the Creative Commons Attribution License, which permits unrestricted use, distribution, and reproduction in any medium, provided the original work is properly cited.

A high-performance silicon UV photodetector was achieved by using a hybrid of a film with nanowires. Electrically conductive and optically transparent indium-tin oxide (ITO) was deposited to form an ITO film or ITO nanowire (NW) on a Si substrate, resulting in a heterojunction. The ITO-film device is stable with a low-leakage current. Meanwhile, the ITO NWs demonstrated an excellent capability to collect photogenerated carriers. The hybrid ITO (NWs on a film)/Si photodetector demonstrates a fast UV reactive time of $1.6 \mathrm{~ms}$ among Si-based photodetectors. We may find a means of enhancing the photoelectric performance capabilities of devices beyond the limits of conventional $\mathrm{Si}$ via the adoption of functional designs. Moreover, the use of a homogeneous material for the structuring of films and nanowires would offer a remarkable advantage by reducing both the number of fabrication steps and the cost.

\section{Introduction}

Si-based devices have been widely utilized in various applications, such as solar cells, LEDs, and photodetectors [1-4]. Especially for photodetectors, rapid response performance is crucial for the realization of sensor networks and optical communications. In addition, high-performing UV photodetectors have specific applications in ozone sensing, flame detection, and military surveillance $[5,6]$. To improve the performance of a UV photodetector, various designs and materials have been applied, such as the GaN-based Schottky junction [6], graphene-based junction [6-8], GaAs/AlGaAs nanowires [9], ITO nanodomes [10], ITO nanowires with $\mathrm{ZnO}$ film $[11,12], \mathrm{NiO} / \mathrm{ZnO}$ films [5], $\mathrm{ZnO} / \mathrm{ZnS}$ nanowires [13], ZnO nanorods [14], ZGO nanowires [15], and Si/ZnO nanowires [1]. When considering the complexity of elaborate photodetector designs, the fabrication steps and cost should also be considered for practical applications [2].

In the cost and fabrication aspects, the functional use of a transparent layer is highly desired to enhance the performance for short-wavelength light while passing the visible-range light. The metal oxide materials have a wide bandgap [5], and thus the highly energetic UV photons can be absorbed. However, the visible-range photons spontaneously pass through the metal oxide layer. This feature renders strong benefits for multifunctional photoelectric devices. Moreover, the active adoption of the metal oxide materials would provide the optical transparency and electrical excellence which are promising advantages for Si-based photoelectric applications. Recently, the significant improvement of UV photodetectors has been realized by using 2D materials and advanced design schemes [16-18].

Here, we report the enhanced performance of Si-based photodetectors using a functional ITO hybrid window. Electrically conductive ITO layers were formed on a $\mathrm{Si}$ substrate to establish a high-quality heterojunction device. In order to maximize the advantages of ITO materials, ITO nanowires were formed on the ITO film, providing significantly enhanced current flows with a less noisy current. This is likely the first report of the use of homogeneous 
materials for the nanowire-embedded film structure applied to a photodetector.

\section{Experimental Procedures}

The $200 \mu \mathrm{m}$-thick FZ p-type Si wafers (100) having resistivity values of $1-3 \Omega \cdot \mathrm{cm}$ were used as substrates and cleaned by acetone, methanol, and DI water under an ultrasonication cleaning process. A rapid thermal annealing process was applied to clean the Si surface and form a thin oxide layer. The thin oxide layer is effective for reducing the reverse saturation current and thus improves the device property [19]. Silicon was used with a thin oxide layer, which is the same for all the devices [20]. Therefore, the observed changes are only due to the top ITO layer. In order to form a heterojunction, a transparent ITO layer was deposited onto the thin oxide-coated $\mathrm{p}$-Si substrate by using a sputter system (SNTEK, Korea). To tailor the ITO layers, various ITO deposition processes were applied to grow the ITO film, ITO nanowires (NWs), and hybrid ITO structure (ITO-NWembedded ITO film). To deposit the ITO film, $100 \mathrm{~W}$ of DC power was applied to an ITO target $\left(\mathrm{In}_{2} \mathrm{O}_{2}\right.$ containing $10 \mathrm{wt} \% \mathrm{SnO}_{2}$ ) for $20 \mathrm{~min}$ at $600^{\circ} \mathrm{C}$.

In order to grow ITO NWs, a thin Ni film $(5 \mathrm{~nm})$ was deposited before the sputtering of the ITO $[11,21,22]$. Preannealed $\mathrm{Ni}$ dots serve as seeds to grow ITO nanowires by the sputtered ITO nanoparticles. The ITO sputtering is almost identical to that of the ITO film growth.

For the hybrid structure of the ITO-NW-embedded ITO film, the ITO film was initially deposited under typical sputtering conditions for $20 \mathrm{~min}$, and the ITO nanowire growth condition was then applied for $8 \mathrm{~min}$. Subsequently, Al metal contacts were formed on the front and back of the photodetector using the same sputtering system. The general configuration of the photodetector is as follows: Al-front/ ITO-window/p-Si/Al_back contact.

A field emission transmission microscope (FETEM, JEOL, JEM-2100F) was used to observe the ITO structures and the interface with the Si substrates. All ITO structures were also formed on glass substrate to monitor the transmittance profiles. Optical transparency was obtained by using a UV-visible spectrophotometer (Shimadzu, UV-1800) for the various ITO structures in the range of $300-1100 \mathrm{~nm}$. Room temperature dark $I-V$ measurements were performed using a source meter unit (Keithley, 2400). Photoresponses of the ITO-embedded Si photodetectors were performed under UV-light illumination from a $400 \mathrm{~nm}$ monochromatic LED lamp. The LED source was calibrated with a power meter (Kusam-Meco, KM-SPM-11). A field emission transmission electron microscope (FETEM, JEOL, JEM-2100F) was used to observe the ITO structures and the interface to Si substrates.

\section{Results and Discussions}

Various types of ITO structures were applied to Si substrates to realize the transparent window-embedded Si photodetectors. In this case, n-type ITO layers spontaneously form a heterojunction by coming into contact with the thin oxide- coated p-type Si substrates. Figure 1(a) shows a schematic of the ITO-embedded Si photodetector. Three different types of ITO structures were applied. A typical ITO film was applied as a reference device. The ITO NW device was prepared by controlling the surficial morphology. The hybrid structure has ITO nanowires grown onto the ITO film. Hereafter, each sample is referred to as the ITO film, ITO NW, and hybrid-ITO. Figure 1(b) shows photograph images of the three devices.

In order to investigate the transmittance, glass samples were also prepared under identical ITO deposition conditions. The transmittance profiles were obtained in the wavelength $(\lambda)$ range of 300 and $1100 \mathrm{~nm}$, as shown in Figure 1(c). The ITO film has the highest average transmittance of $86.72 \%$. The ITO film has an oscillation feature due to the light interference of the ITO-film layer. Meanwhile, no significant oscillation profiles were observed from the ITO-NW structure or hybrid-ITO window samples due to the existing nanoscale entities to relieve the light interference.

Meanwhile, the ITO-NW sample has a lower transmittance value of $73.40 \%$ due to light scattering by the nanowires [15]. For the hybrid-ITO sample, the combination of ITO film and ITO nanowires reduced the transmittance value further by $69.88 \%$. According to the transmittance profiles, nanowire-embedded surfaces have an overall lower transmittance value for short wavelengths, which clearly indicates the strong interaction between nanowires and short $\lambda$ photons.

SEM observation was employed to show the structural changes of the samples. Figure 2(a) shows the top-view image of the ITO thin film deposited on a Si substrate, which confirms the uniform growth. The film thickness is approximately $180 \mathrm{~nm}$, as shown in Figure 2(d). Meanwhile, the sample grown with ITO nanowires (ITO NWs) has a relatively thin ITO base film of approximately $130 \mathrm{~nm}$ (Figure 2(e)). A single ITO NW has a Ni tip on the top of the ITO NW, which is an intrinsic feature of the ITO NW grown by the sputtering method [21].

Figure 2(b) depicts regular ITO nanowires on an ITO base film. The hybrid-ITO sample is presented in Figure 2(c), showing the combined structures of ITO nanowires and the ITO film. Due to the combined processes of the ITO-film formation step and the ITO-nanowire growth step, the ITO base film has a thickness of $227 \mathrm{~nm}$, as shown in Figure 2(f). Short nanowires and nanoscale clusters can clearly be observed in Figure 2(c).

The working mechanism of the heterojunction device is according to the energy band diagram of Figure 3(c). The type II junction is suitable for drifting photogenerated carriers to external electrodes. In order to determine the work function (WF) of ITO, Kelvin probe force microscopy (KPFM) measurement was performed. The topography and the WF map of ITO are shown in Figures 3(a) and 3(b), respectively. The topography FESEM image is shown in Figure 2 of the ITO surface and the corresponding root mean square (RMS) profile was calculated to be $1.76 \mathrm{~nm}$. Meanwhile, the WF of ITO was obtained as $4.71 \mathrm{eV}$.

To characterize the device performances, current-voltage profiles were obtained for all three devices under a dark 


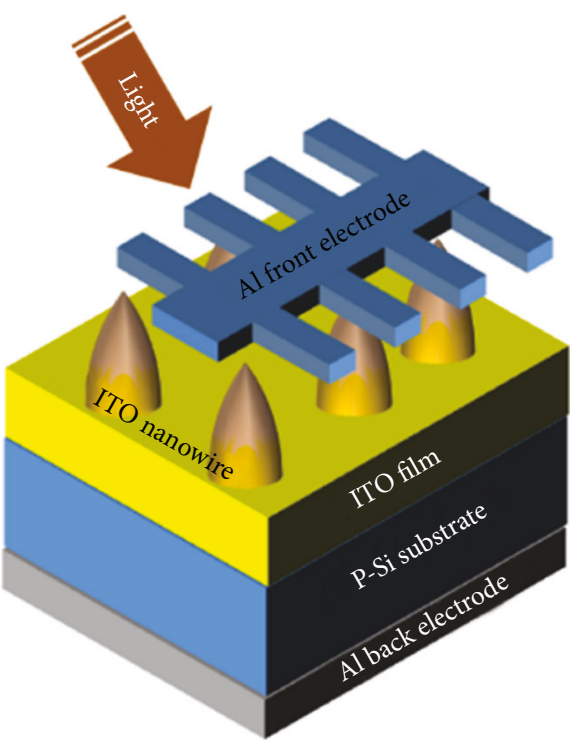

(a)

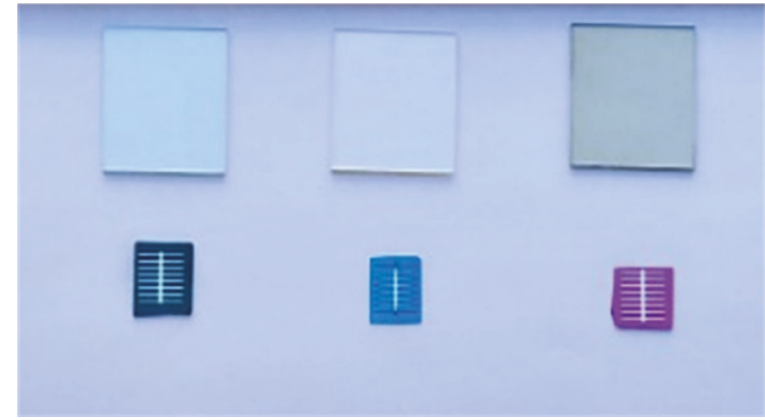

(b)

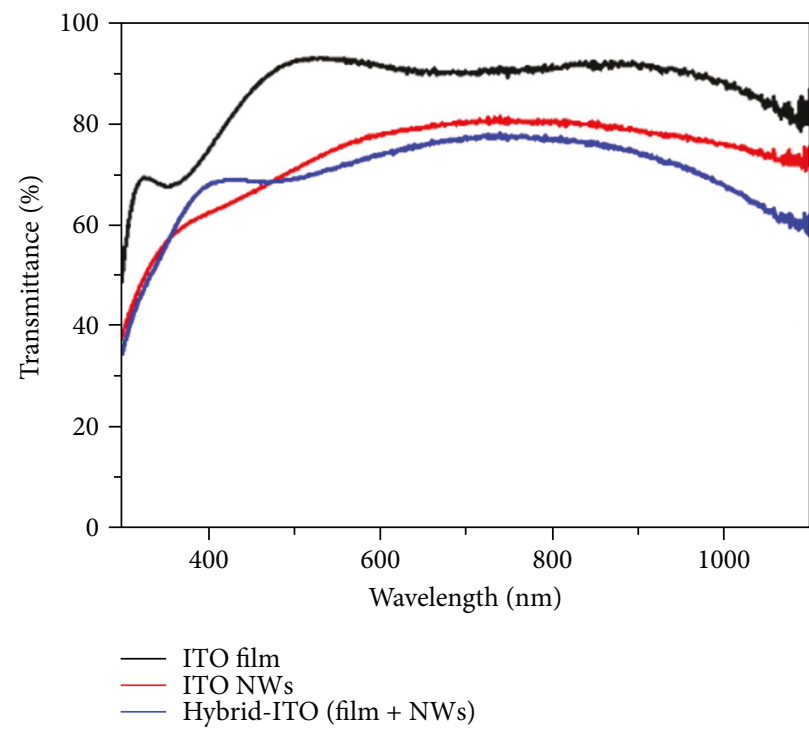

(c)

FIGURE 1: (a) Schematic of the hybrid-ITO (ITO-nanowire-embedded ITO film) photodetector, (b) photographic images of Si photodetectors and ITO-coated glasses, and (c) transmittance profiles of the ITO-film, ITO-NW, and hybrid-ITO window samples.

condition, as shown in Figure 4. In order to evaluate the rectifying characteristics, the rectification ratios were obtained by the ratio of the current value of $1.5 \mathrm{~V}$ to the current value of $-1.5 \mathrm{~V}$. The ITO-film device provides a good rectification ratio of 161.37. Meanwhile, the ITO-nanowire device has a lower value of 7.167. This is mainly caused by the reverse saturation current density of $9.21\left(\mathrm{~mA} / \mathrm{cm}^{2}\right)$, which is 35 times higher than $0.256\left(\mathrm{~mA} / \mathrm{cm}^{2}\right)$ of the ITO-film device. Interestingly, a significantly enhanced rectification value of 891.69 was obtained from the hybrid-ITO structures. This improvement was induced due mainly to the improved forward current density, which is more than double to that of the ITO-film device (Table 1). Moreover, the reverse saturation current was also reduced, which clearly reflects the inherent benefit of the ITO film.
To qualify the diode junction, the ideality factor $(n)$ was obtained from the following equation:

$$
n=\frac{q}{k T} \frac{\partial V}{\partial(\ln I)}=\frac{q}{k T} \frac{\partial V}{2.3 \times \partial(\log I)},
$$

where $q, k T$, and $I$ are the electron charge, thermal energy $(\mathrm{eV})$, and current, respectively. The ITO-film device has a fair value of 1.73 , compared to the ITO-NW device which has a value of 1.92. On the other hand, an improved value (1.25) was achieved from the hybrid-ITO device, which confirms a better-quality junction compared to that of the ITO-film device (Table 1). The ITO-NW surface effectively provides a carrier transport route from $\mathrm{Si}$ to the Al-front contact to resolve the current spreading effect from the conventional 


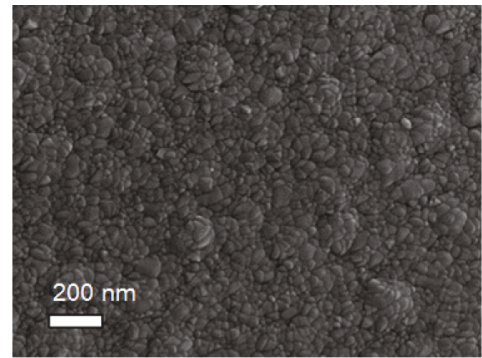

(a)

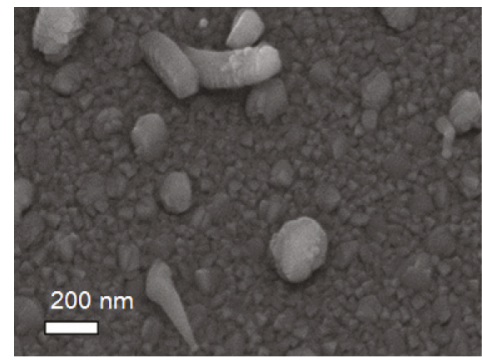

(c)

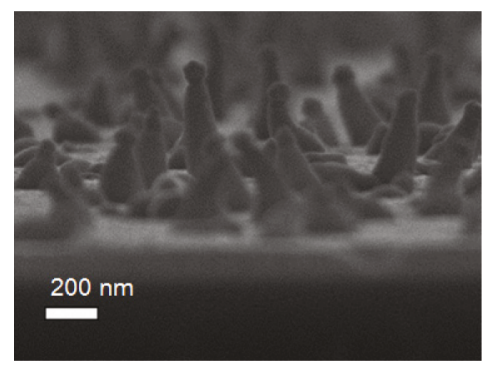

(e)

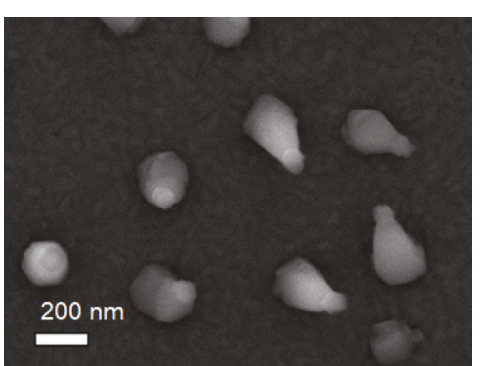

(b)

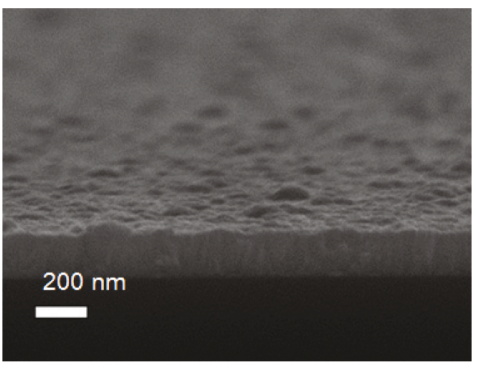

(d)

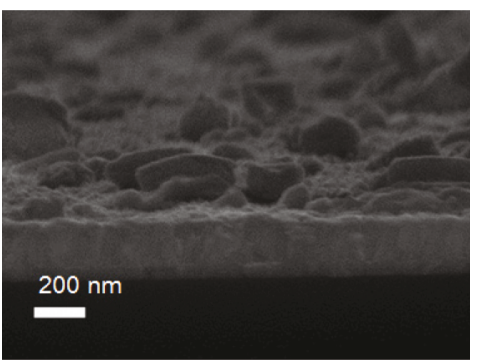

(f)

FIgURE 2: Top-view and cross-sectional-view of FESEM images of (a,d) ITO-film, (b,e) ITO-NW, and (c,f) the hybrid-ITO (ITO-NWembedded ITO film) samples.

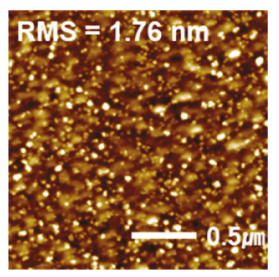

(a)
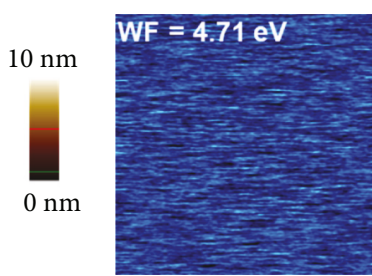

(b)

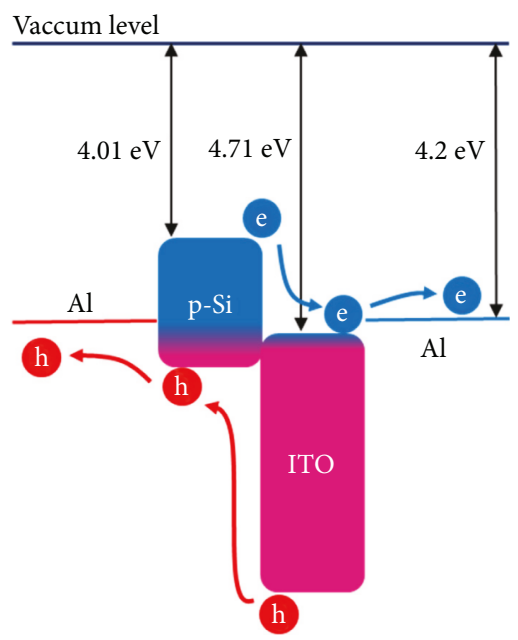

(c)

FIGURE 3: ITO (a) topography, (b) work function map, and (c) energy band diagram of the Al/ITO/P-Si/Al device.

ITO-film conductor [10]. The conventional ITO conductor has a disadvantage for electron movements due to the film shape for the current spreading effect. Meanwhile, the sharp geometric feature of ITO NWs spontaneously provides the point contact to the Al-front metal to induce an efficient flow of electrons. The hybrid structure simultaneously has 


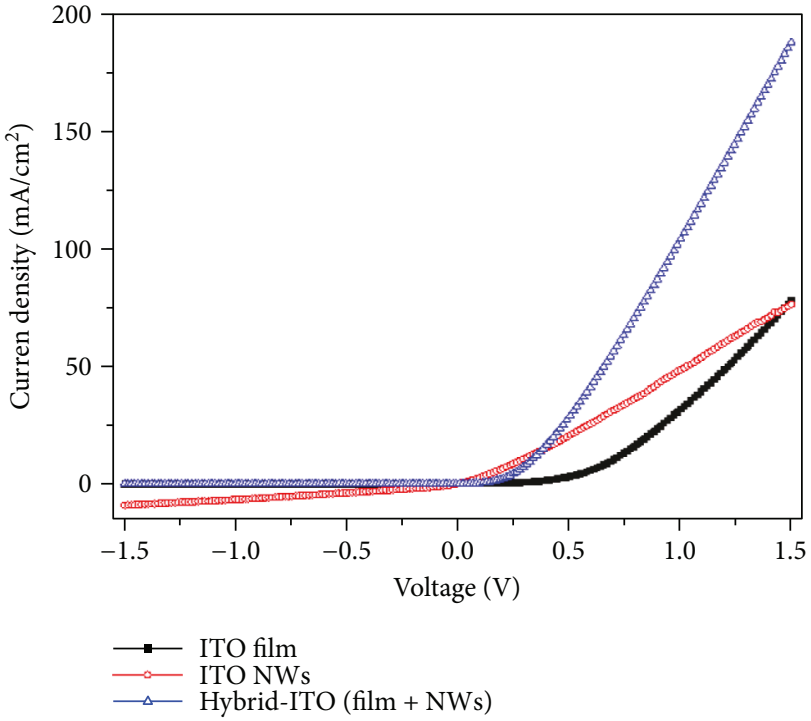

(a)

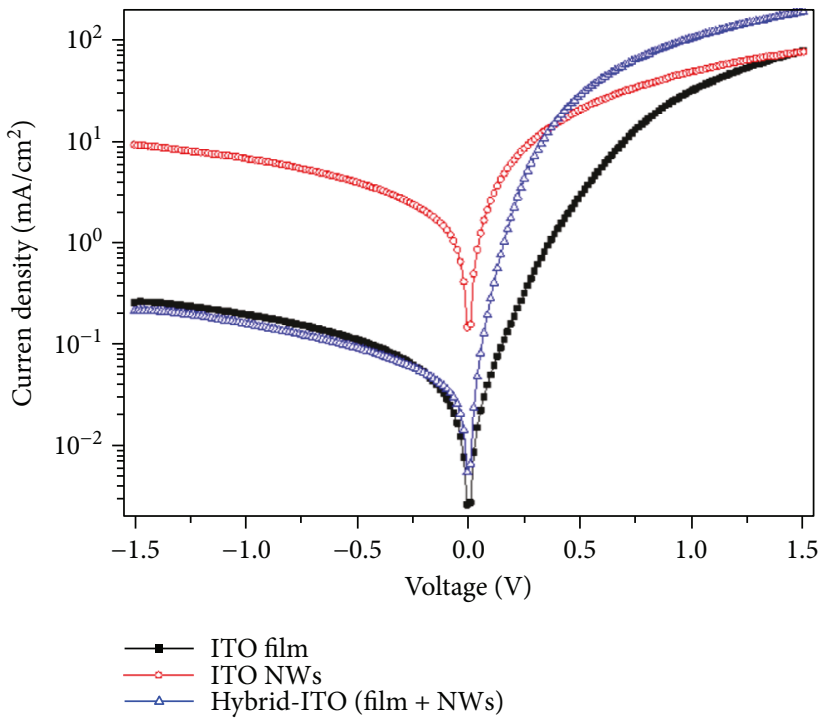

(b)

FIgURE 4: $I-V$ characteristics of the ITO-film device, ITO-NW device, and the hybrid-ITO (ITO-nanowire-embedded ITO film) device. (a) Linear plot and (b) semilog plot.

TABLE 1: Performance of the ITO-film device, ITO-NW device, and the hybrid-ITO (ITO-nanowire-embedded ITO film) device.

\begin{tabular}{lccc}
\hline & ITO film & ITO NWs & Hybrid-ITO \\
\hline Current at $+1.5 \mathrm{~V}\left(\mathrm{~mA} / \mathrm{cm}^{2}\right)$ & 0.0779 & 0.0762 & 0.188 \\
Current at $-1.5 \mathrm{~V}\left(\mathrm{~mA} / \mathrm{cm}^{2}\right)$ & -0.256 & -9.21 & 0.211 \\
$\quad$ Rectifying ratio & 161.37 & 7.167 & 891.69 \\
$\quad$ Ideality factor & 1.73 & 1.92 & 1.25 \\
Response ratio $\left(I_{\mathrm{UV}-\mathrm{On}} /\right.$ & 4.85 & 1.194 & 7.972 \\
$\left.I_{\mathrm{UV}-\text { Off }}\right)$ & -7.27 & -8.44 & -9.84 \\
$I_{\mathrm{UV}-\text { On }}(\mu \mathrm{A})$ & -1.50 & -7.07 & -1.23 \\
$I_{\mathrm{UV}-\text { Off }}(\mu \mathrm{A})$ & 0.00895 & 0.00215 & 0.0016 \\
$\mathrm{UV}$-reactive time $\left(\tau_{\mathrm{UV}-\mathrm{On}}\right)(\mathrm{s})$ & 0.0004 & 0.0004 & 0.0003 \\
Recovery time $\left(\tau_{\mathrm{UV}-\mathrm{Off}}\right)(\mathrm{s})$ & & & \\
\hline
\end{tabular}

advantages for the quality junction formation in the merit of the ITO film and effective collection formation due to the ITO NWs.

In order to investigate the photoelectric performances, photoresponse measurements were obtained from all three samples. The photoresponse performances were obtained by turning on and off the UV light illumination. The UV LED source has a wavelength of $400 \mathrm{~nm}$ with a light intensity of $3 \mathrm{~mW} / \mathrm{cm}^{2}$. By illuminating the UV light, significant responses were clearly obtained from all three samples, as shown in Figure 5. To estimate the photoreactive performances, the photoresponse ratio (PR) was obtained by the following relation:

$$
\text { Photoresponse ratio }=\left|\frac{I_{\mathrm{UV} \text {-on }}}{I_{\mathrm{UV} \text {-of f }}}\right| \text {. }
$$

The PR is a function of the light-induced current over the dark current. The ITO-NW device has the lowest value of 1.194 , which is attributed to the high dark current $\left(I_{\mathrm{UV}-\mathrm{Off}}\right)$ value of $-7.07 \mu \mathrm{A}$, compared to the light current $\left(I_{\mathrm{UV}-\mathrm{On}}\right)$ value of $-8.44 \mu \mathrm{A}$. This serious dark current is induced from the poor interface between Si and ITO NWs. Meanwhile, the ITO-film device has an improved PR value of 4.85 by the suppressed $I_{\text {UV-Off }}$ value $(-1.50 \mu \mathrm{A})$ and a much enhanced $I_{\mathrm{UV}-\text { On }}$ value $(-7.27 \mu \mathrm{A})$. A remarkable $\mathrm{PR}$ value of 7.97 was obtained from the hybrid-ITO device. This result signifies the combined advantages of the ITO-film device and the ITO-NW device. The ITO nanowires have a tapered feature with $\mathrm{Ni}$ tips, which induce the efficient flows of the photogenerated carriers, resulting in a remarkably improved $I_{\text {UV-On }}$ value $(-9.86 \mu \mathrm{A})$. In addition, the ITO film provides a high-quality interface to $\mathrm{Si}$ and effectively controls the $I_{\text {UV-Off }}$ value $(-1.23 \mu \mathrm{A})$.

The photodetection performances of the three devices were evaluated by the response speed. The UV reactive time $\left(\tau_{\mathrm{UV}-\mathrm{On}}\right)$ and UV recovery time $\left(\tau_{\mathrm{UV}-\mathrm{Off}}\right)$ were estimated. The change of current $\left(I_{\text {change }}\right)$ was obtained by the following equation:

$$
I_{\text {change }}=I_{\mathrm{UV}-\mathrm{On}}-I_{\mathrm{UV}-\mathrm{Off}} \text {. }
$$

The $\tau_{\mathrm{UV}-\mathrm{On}}$ is defined as the time taken for the increase of the current value from $10 \%$ to $90 \%$ of the change of current [11]. The $\tau_{\mathrm{UV}-\mathrm{Off}}$ is the time for the decrease of the current value from $90 \%$ to $10 \%$ of the change of current. Due to the abrupt heterojunction of the ITO and Si contact, the $\tau_{\mathrm{UV}-\text { Off }}$ values are excellent $(0.3-0.4 \mathrm{~ms})$ for all three devices, as presented in Figure 4(b). In the aspects for UV reaction, different huge performances appeared by ITO structures. The ITO-NW device shows a fast UV response time of $2.15 \mathrm{~ms}$. Compared to this, the ITO film has the longer 


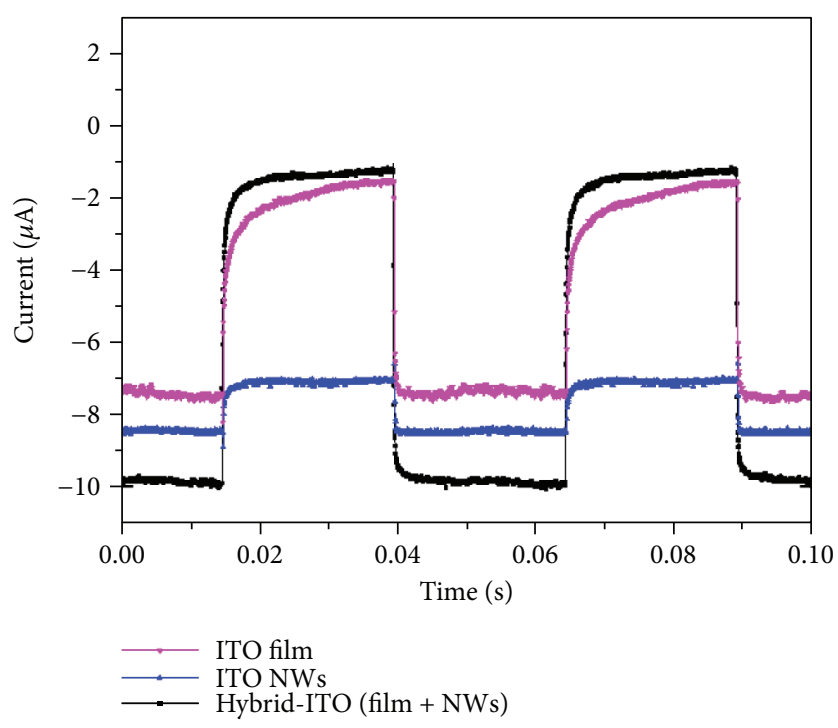

(a)

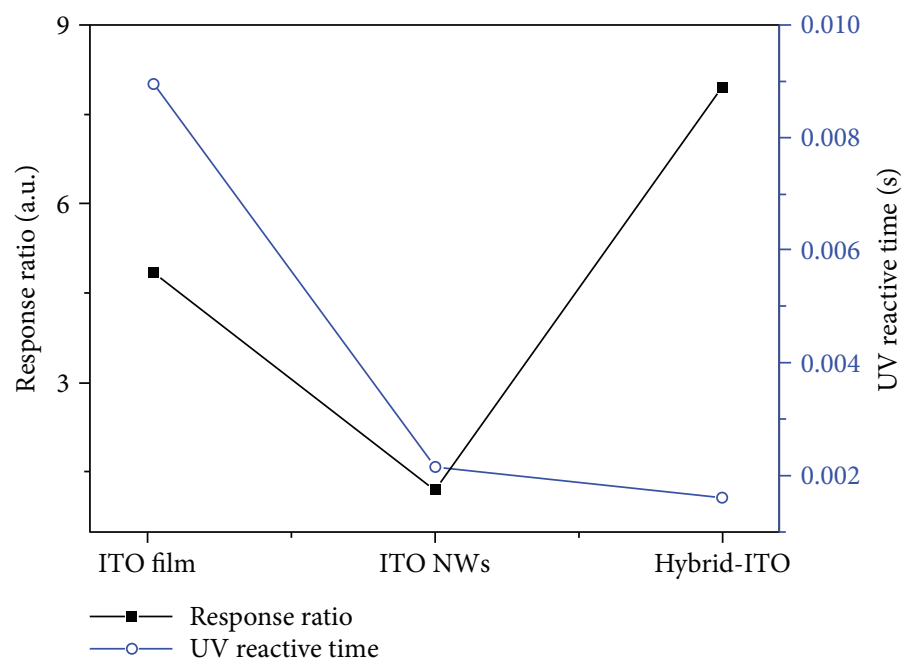

(b)

FIgure 5: Photoresponses of the ITO-film device, ITO-NW device, and the hybrid-ITO (ITO-nanowire-embedded ITO film) device. (a) Current-time profiles. (b) Response ratio and UV-reactive time plots.

$\tau_{\text {UV-On }}$ time of $8.95 \mathrm{~ms}$, which signifies the delay of the collection time for the photogenerated carriers. Due to the film type, the photogenerated carriers readily spread through the ITO film. This is due to the similar resistance values between the ITO film and the Al-metal contact [10]. A significantly improved result was obtained from the hybrid-ITO device for the $\tau_{\text {UV-On }}$ value of $1.6 \mathrm{~ms}$, which outperforms other Si-based photodetectors $[4,11,23,24]$ and is a further enhancement over organic devices [25].

These results strongly suggest that the photoelectric device performances can be improved by doing structural engineering [26]. In this work, the hybrid-ITO structure (ITO-NW-embedded ITO film) demonstrated significantly enhanced device performances. This is a promising advantage with regard to device fabrication using homogenous materials with consecutive processes, in contrast to heterogeneous devices which require multiple and complex processes.
We may find a route by which to realize efficient photoelectric devices by adopting the advantages of the nanostructures and films described here.

\section{Conclusion}

Optically transparent and electrically conductive ITO was applied to form a quality ITO film or ITO nanowires for high-performance Si UV photodetectors. Due to the utilization of a homogeneous material for the ITO film and ITO nanowires, an identical sputtering method can be applied to tailor the ITO front surface onto a Si substrate, which is an affordable device fabrication approach which does not interrupt the device processes or lead to complex issues. The hybrid-ITO (nanowire-embedded ITO film) device showed greatly enhanced UV photoresponses compared to the film consisting of only ITO or the ITO nanowire device. A 
remarkably fast UV reactive time of $1.6 \mathrm{~ms}$ was obtained due to the combined merits of the nanowires and film.

Homogeneous material-based designs are rarely reported for photoelectric applications. However, the effect is substantial with affordable processing steps. In the future, we may find a strong design approach for functional photoelectric devices with unexpected but substantial enhancements.

\section{Data Availability}

The data used to support the findings of this study are available from the corresponding author upon request.

\section{Conflicts of Interest}

The authors declare that they have no conflicts of interest.

\section{Authors' Contributions}

Hyunki Kim and Gyeong-Nam Lee contributed equally to this work.

\section{Acknowledgments}

This research was supported by Basic Science Research Program through the National Research Foundation (NRF) of Korea by the Ministry of Education (NRF2018R1D1A1B07045336) and the Korea Institute of Energy Technology Evaluation and Planning by the Ministry of Knowledge Economy (KETEP-20168520011370).

\section{References}

[1] Z. Wang, R. Yu, X. Wen et al., "Optimizing performance of silicon-based $\mathrm{p}-\mathrm{n}$ junction photodetectors by the piezophototronic effect," ACS Nano, vol. 8, no. 12, pp. 1286612873, 2014

[2] S. Tallur and S. A. Bhave, "A silicon electromechanical photodetector,” Nano Letters, vol. 13, no. 6, pp. 2760-2765, 2013.

[3] L. Shi and S. Nihtianov, "Comparative study of silicon-based ultraviolet photodetectors," IEEE Sensors Journal, vol. 12, no. 7, pp. 2453-2459, 2012.

[4] D. K. Lee, H. Ko, and Y. Cho, "Single Si submicron wire photodetector fabricated by simple wet etching process," Materials Letters, vol. 160, pp. 562-565, 2015.

[5] M. Patel, H.-S. Kim, H.-H. Park, and J. Kim, "Active adoption of void formation in metal-oxide for all transparent superperforming photodetectors," Scientific Reports, vol. 6, no. 1, article 25461, 2016.

[6] W.-Y. Kong, G.-A. Wu, K.-Y. Wang et al., "Graphene- $\beta$ $\mathrm{Ga}_{2} \mathrm{O}_{3}$ heterojunction for highly sensitive deep UV photodetector application," Advanced Materials, vol. 28, no. 48, pp. 10725-10731, 2016.

[7] F. Liu and S. Kar, "Quantum carrier reinvestment-induced ultrahigh and broadband photocurrent responses in graphene-silicon junctions," ACS Nano, vol. 8, no. 10, pp. 10270-10279, 2014.

[8] S. Schuler, D. Schall, D. Neumaier et al., "Controlled generation of a $\mathrm{p}-\mathrm{n}$ junction in a waveguide integrated graphene photodetector," Nano Letters, vol. 16, no. 11, pp. 7107-7112, 2016.
[9] X. Dai, S. Zhang, Z. Wang et al., "GaAs/AlGaAs nanowire photodetector," Nano Letters, vol. 14, no. 5, pp. 26882693, 2014.

[10] S.-H. Hong, J.-H. Yun, H.-H. Park, and J. Kim, "Nanodome-patterned transparent conductor for highly responsive photoelectric device," Applied Physics Letters, vol. 103, no. 15, article 153504, 2013.

[11] H. Kim, M. D. Kumar, M. Patel, and J. Kim, "ITO nanowiresembedding transparent $\mathrm{NiO} / \mathrm{ZnO}$ photodetector," Materials Research Bulletin, vol. 83, pp. 35-40, 2016.

[12] Z. Ke, Z. Yang, M. Wang, M. Cao, Z. Sun, and J. Shao, "Low temperature annealed $\mathrm{ZnO}$ film UV photodetector with fast photoresponse," Sensors and Actuators A: Physical, vol. 253, pp. 173-180, 2017.

[13] S. C. Rai, K. Wang, Y. Ding et al., "Piezo-phototronic effect enhanced UV/visible photodetector based on fully wide band gap type-II $\mathrm{ZnO} / \mathrm{ZnS}$ core/shell nanowire array," ACS Nano, vol. 9, no. 6, pp. 6419-6427, 2015.

[14] J. Saghaei, A. Fallahzadeh, and T. Saghaei, "Vapor treatment as a new method for photocurrent enhancement of UV photodetectors based on $\mathrm{ZnO}$ nanorods," Sensors and Actuators A: Physical, vol. 247, pp. 150-155, 2016.

[15] J. Wang, C. Yan, M.-F. Lin, K. Tsukagoshi, and P. S. Lee, "Solution-assembled nanowires for high performance flexible and transparent solar-blind photodetectors," Journal of Materials Chemistry C, vol. 3, no. 3, pp. 596-600, 2015.

[16] M. Zhu, L. Zhang, X. Li et al., " $\mathrm{TiO}_{2}$ enhanced ultraviolet detection based on a graphene/Si Schottky diode," Journal of Materials Chemistry A, vol. 3, no. 15, pp. 8133-8138, 2015.

[17] F. X. Liang, J. Z. Wang, Y. Wang et al., "Single-layer graphene/ titanium oxide cubic nanorods array/FTO heterojunction for sensitive ultraviolet light detection," Applied Surface Science, vol. 426, pp. 391-398, 2017.

[18] T. F. Zhang, G. A. Wu, J. Z. Wang et al., “A sensitive ultraviolet light photodiode based on graphene-on-zinc oxide Schottky junction," Nano, vol. 6, no. 5, pp. 1073-1081, 2017.

[19] Y. Song, X. Li, C. Mackin et al., "Role of interfacial oxide in high-efficiency graphene-silicon Schottky barrier solar cells," Nano Letters, vol. 15, no. 3, pp. 2104-2110, 2015.

[20] X. Li, M. Zhu, M. Du et al., "High detectivity graphene-silicon heterojunction photodetector," Small, vol. 12, no. 5, pp. 595601,2016

[21] H. Kim, H.-H. Park, and J. Kim, "Electrical and optical properties of Ni-assisted grown single crystalline and transparent indium-tin-oxide nanowires," Materials Science in Semiconductor Processing, vol. 48, pp. 79-84, 2016.

[22] J.-H. Yun, M. M. D. Kumar, Y. C. Park, and J. Kim, "Transparent conductors with an ultrathin nickel layer for high-performance photoelectric device applications," Materials Science in Semiconductor Processing, vol. 31, pp. 334-339, 2015.

[23] H. Bencherif, F. Djeffal, and H. Ferhati, "Performance enhancement of $\mathrm{Pt} / \mathrm{TiO}_{2} / \mathrm{Si} \mathrm{UV}$-photodetector by optimizing light trapping capability and interdigitated electrodes geometry," Superlattices and Microstructures, vol. 97, pp. 303-312, 2016.

[24] H. Kim, M. D. Kumar, and J. Kim, "Highly-performing Ni/ $\mathrm{SiO}_{2} / \mathrm{Si}$ MIS photodetector for NIR detecting applications," Sensors and Actuators A: Physical, vol. 233, pp. 290-294, 2015. 
[25] H. A. Afify, M. M. el-Nahass, A.-. S. Gadallah, and M. Atta Khedr, "Carrier transport mechanisms and photodetector characteristics of $\mathrm{Ag} / \mathrm{TiOP} / \mathrm{p}$-si/Al hybrid heterojunction," Materials Science in Semiconductor Processing, vol. 39, pp. 324-331, 2015.

[26] V. Gurylev, C. Y. Su, and T. P. Perng, "Hydrogenated $\mathrm{ZnO}$ nanorods with defect-induced visible light-responsive photoelectrochemical performance," Applied Surface Science, vol. 411, pp. 279-284, 2017. 


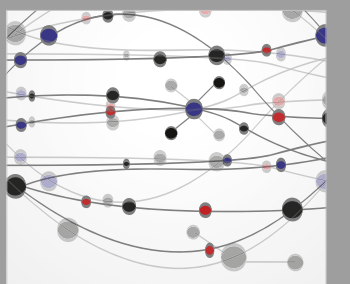

The Scientific World Journal
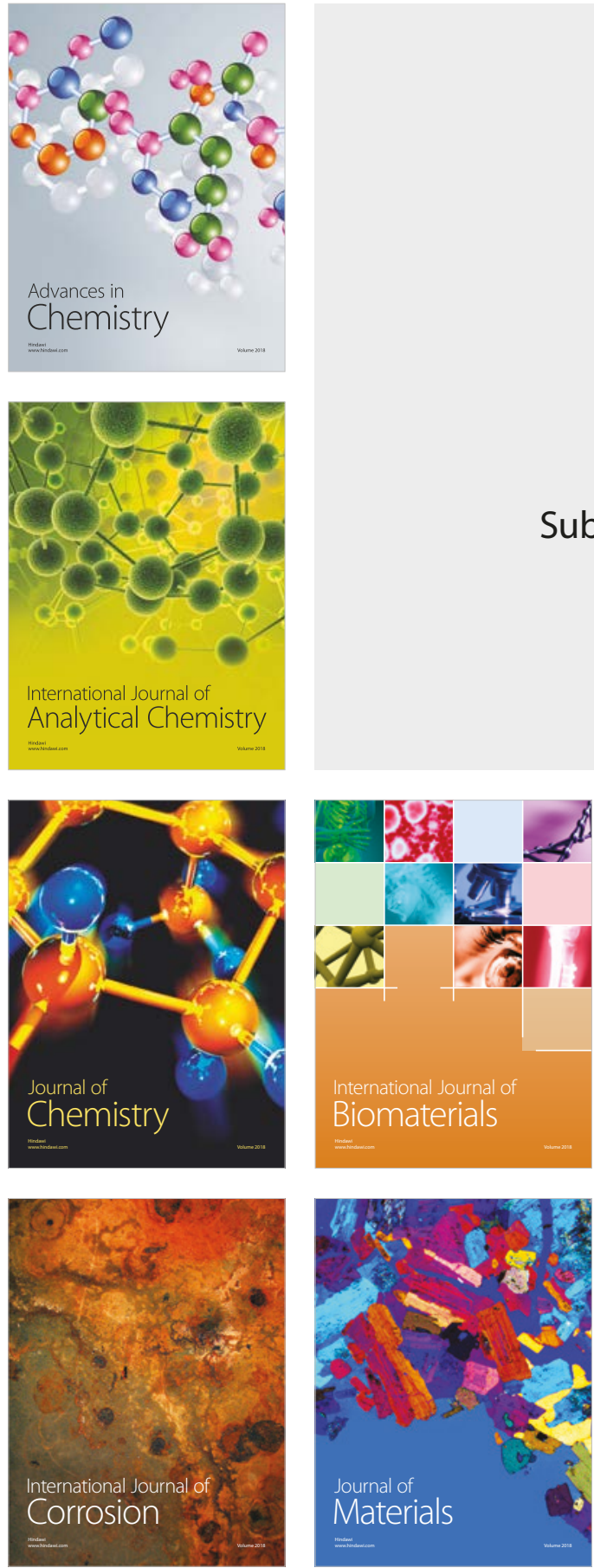

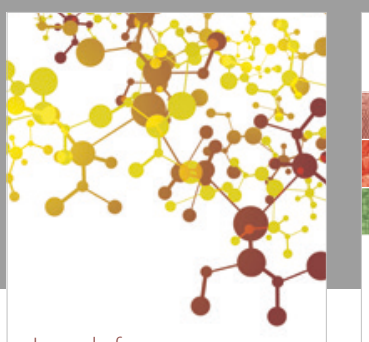

Journal of

Applied Chemistry
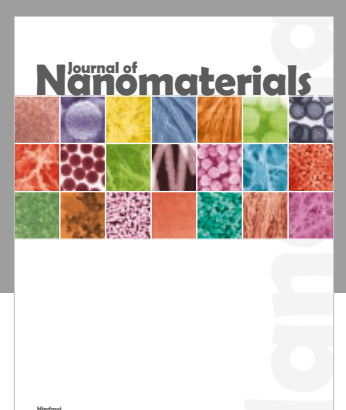

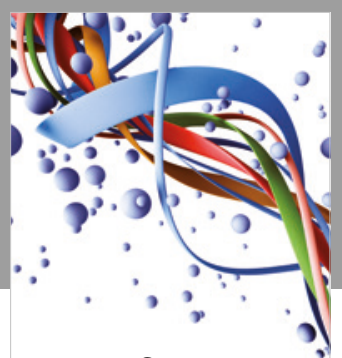

Scientifica

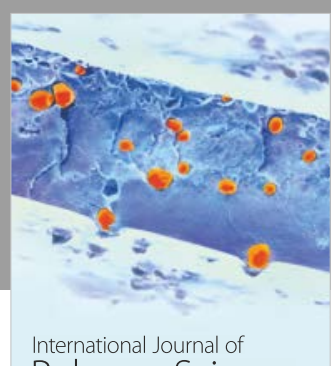

Polymer Science

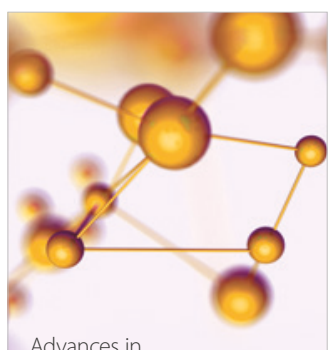

Physical Chemistry
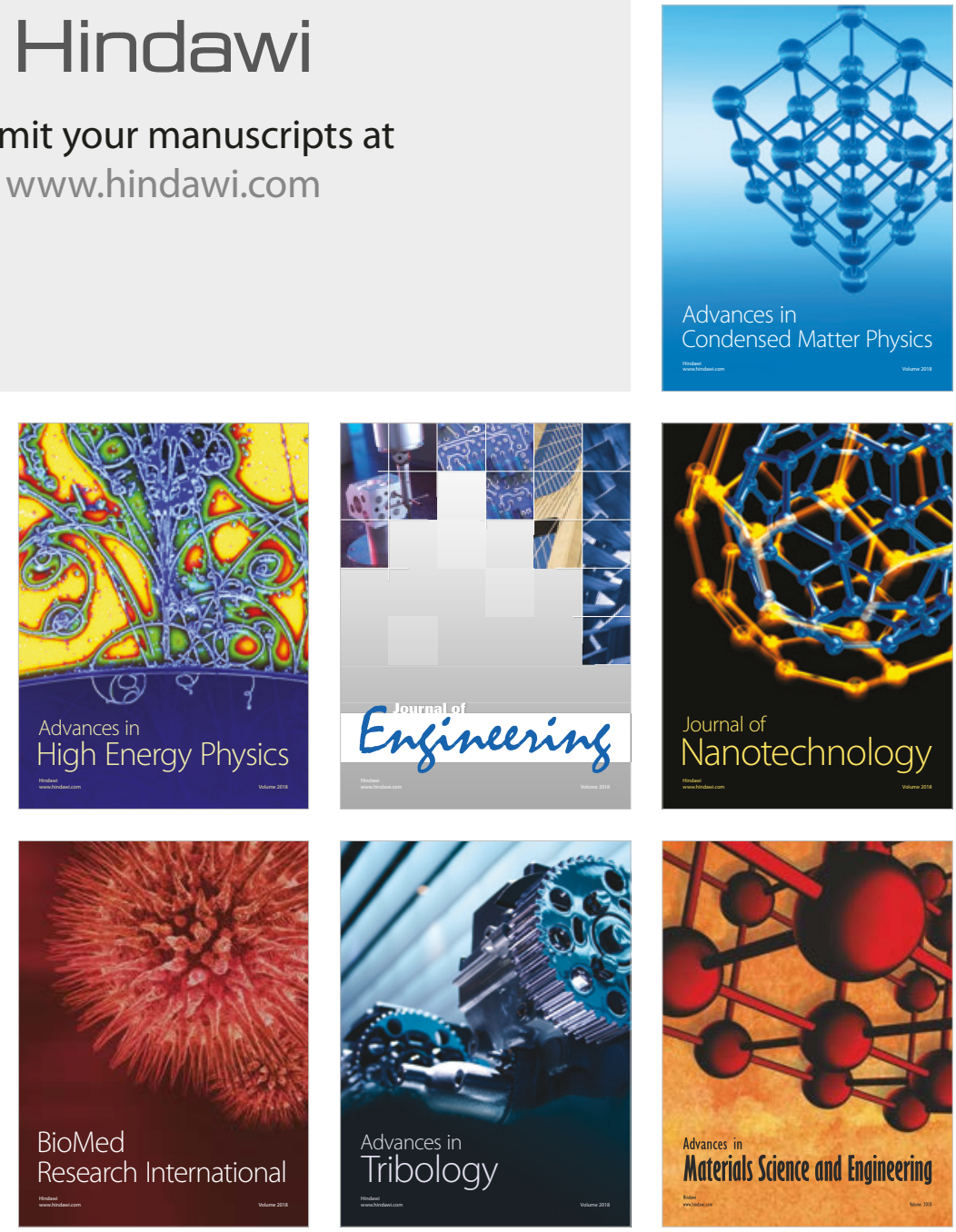